\title{
Chemokine Receptor CXCR2 Activates Distinct Pathways for Chemotaxis and Calcium Mobilization
}

\author{
Futoshi ShIBATA, ${ }^{*, a}$ Kiyoshi Konishi, ${ }^{b}$ and Hideo NAKAGAWA ${ }^{a}$ \\ ${ }^{a}$ Department of Physiological Chemistry, Faculty of Pharmaceutical Sciences, Toyama Medical and Pharmaceutical \\ University; 2630 Sugitani, Toyama 930-0194, Japan: and ${ }^{b}$ Department of Microbiology, Nippon Dental University; \\ 1-9-20 Fujimi, Chiyoda-ku, Tokyo 102-8159, Japan. Received April 25, 2002; accepted June 7, 2002
}

\begin{abstract}
Rat cytokine-induced neutrophil chemoattractant-3 (CINC-3) has neutrophil chemotactic activity comparable with that of CINC-1 and CINC-2, but induces calcium mobilization more potently than CINC-1 and CINC-2. However, only one CINC receptor, CXCR2, has been found in rat neutrophils. Therefore we attempted to determine the biochemical basis for the differences in neutrophil responses to CINC-1/-2 versus CINC-3. Both chemotactic activity and calcium mobilization induced by CINC-3 were desensitized by a 100-fold excess of CINC-1, which was consistent with our previous results showing that CINC-1 has 70-fold lower affinity to the receptor on rat neutrophils than CINC-3. Desensitization appeares to be reflected by the affinity of the ligands to the receptor. CINC-1- and CINC-3-induced chemotaxis was sensitive to inhibition by pertussis toxin, whereas calcium mobilization induced by CINC-1 and CINC-3 was insensitive. These results suggest that CINCs induce neutrophil chemotaxis and calcium mobilization through distinct G-proteins with different efficiency.
\end{abstract}

Key words cytokine-induced neutrophil chemoattractant (CINC); pertussis toxin; chemotaxis; calcium mobilization; macrophage inflammatory protein-2 (MIP-2); chemokine

Cytokine-induced neutrophil chemoattractants (CINCs) are potent neutrophil chemotactic factors in rats and major mediators of acute inflammation. ${ }^{1-3)}$ Four members of the CINC family have been identified: CINC-1, CINC- $2 \alpha$, CINC-2 $\beta$, and CINC-3/macrophage inflammatory protein- 2 (MIP-2) ${ }^{1)}$ CINC-2 $\alpha$ and CINC-2 $\beta$ differ only in the sequence of carboxy-terminal three amino acid residues and are produced by alternative splicing. ${ }^{4)}$ CINCs show high degrees of sequence similarity to human GROs, interleukin 8 (IL-8), mouse KC, and mouse MIP-2 and belong to the CXC chemokine family. CINC-1 and CINC-2 show chemotactic activity comparable with that of CINC-3. ${ }^{5)}$ Treatment of neutrophils with CINC-1 or CINC-2 inhibited the chemotactic activity of CINC-1 and CINC-2, but not that of CINC-3, whereas treatment with CINC-3 inhibited the activity of all CINCs. $\left.{ }^{6}\right)$ On the other hand, CINC-3 induced calcium mobilization more potently than CINC-1 and CINC-2, and desensitized calcium mobilization in neutrophils induced by CINC-1 and CINC-2. ${ }^{5)}$ In addition, CINC-3 potently competed with the binding of ${ }^{125}$ I-labeled CINC-3 Tyr, although CINC-1 competed only weakly. ${ }^{7}$ To determine the biochemical basis for the differences in neutrophil responses to CINC$1 /-2$ versus $\mathrm{CINC}-3$, we have cloned the gene encoding the receptor and identified rat CXCR2 as the common receptor for all CINCs. ${ }^{8)}$ CXCR2 belongs to the family of G-proteincoupled receptors with seven transmembrane-spanning domains. Herein, we report the relationship between the biological activities of CINCs and G-protein usage.

\section{MATERIALS AND METHODS}

Preparation of Neutrophils Neutrophils were harvested from the peritoneal exudate $16 \mathrm{~h}$ after intraperitoneal injection of Krebs/Ringer bicarbonate solution containing $1 \%$ casein $(120 \mathrm{ml} / \mathrm{kg}$ body weight of male Wistar rats), washed twice with Gey's balanced salt solution and twice with the assay buffer and then resuspended at a concentration of $10^{7}$ cells $/ \mathrm{ml}^{5}{ }^{5}$
Pertussis Toxin Treatment Neutrophils were incubated with pertussis toxin (PTX, $1 \mu \mathrm{g} / \mathrm{ml}$ ) at $37^{\circ} \mathrm{C}$ for $2 \mathrm{~h}$ in a humidified $5 \% \mathrm{CO}_{2}$ atmosphere and washed twice with the assay buffer.

Measurement of Intracellular Free Calcium Levels The intracellular free calcium level in neutrophils was measured by the method described previously. ${ }^{5)}$ Neutrophils were incubated with the calcium indicator fura-2 acetoxymethyl ester $(2.5 \mu \mathrm{M})$ in $\mathrm{Ca}^{2+} / \mathrm{Mg}^{2+}$-free phosphate-buffered saline containing $10 \mathrm{~mm}$ HEPES, $0.25 \%$ bovine serum albumin, and $10 \mathrm{~mm}$ glucose $(\mathrm{pH} 7.4)$ for $30 \mathrm{~min}$ at $37^{\circ} \mathrm{C}$. After incubation, the cells were washed twice and resuspended at $10^{6}$ cells $/ \mathrm{ml}$ with the buffer. In the presence of $1 \mathrm{mM} \mathrm{CaCl}_{2}$, fluorescence changes were monitored using a Hitachi F4500 fluorescence spectrophotometer at excitation wavelengths of 340 and $380 \mathrm{~nm}$ and an emission wavelength of $510 \mathrm{~nm}$. Fura-2loaded cells were stimulated with CINCs, then lysed with $0.1 \%$ Triton-X, and finally $\mathrm{Ca}^{2+}$ was chelated with $6 \mathrm{~mm}$ EGTA. Intracellular calcium levels were calculated as described by Grynkiewicz et al. ${ }^{9)}$

Chemotaxis Assay in Vitro Chemotactic activity for rat neutrophils was assayed in 96-well plates as described previously. ${ }^{5)}$ As an index of chemotaxis, the migration rate was expressed as the percentage of the number of neutrophils migrating into the lower wells during an 80-min incubation to that of the neutrophils applied to the upper wells.

\section{RESULTS}

Rat CXCR2 on neutrophils is the unique receptor for all three CINCs. We determined whether the biological activities of CINC-3, a potent agonist, were desensitized by CINC-1. First, the effects of desensitization of CINCs on calcium mobilization were examined in rat neutrophils. Neutrophils exposed first to CINC-1 at $100 \mathrm{~nm}$ became unresponsive to subsequent stimulation with CINC-1 at the same concentration, but a second stimulation at a higher concentration $(1 \mu \mathrm{M})$ caused reduced calcium mobilization (Figs. 1A, B). CINC-3 

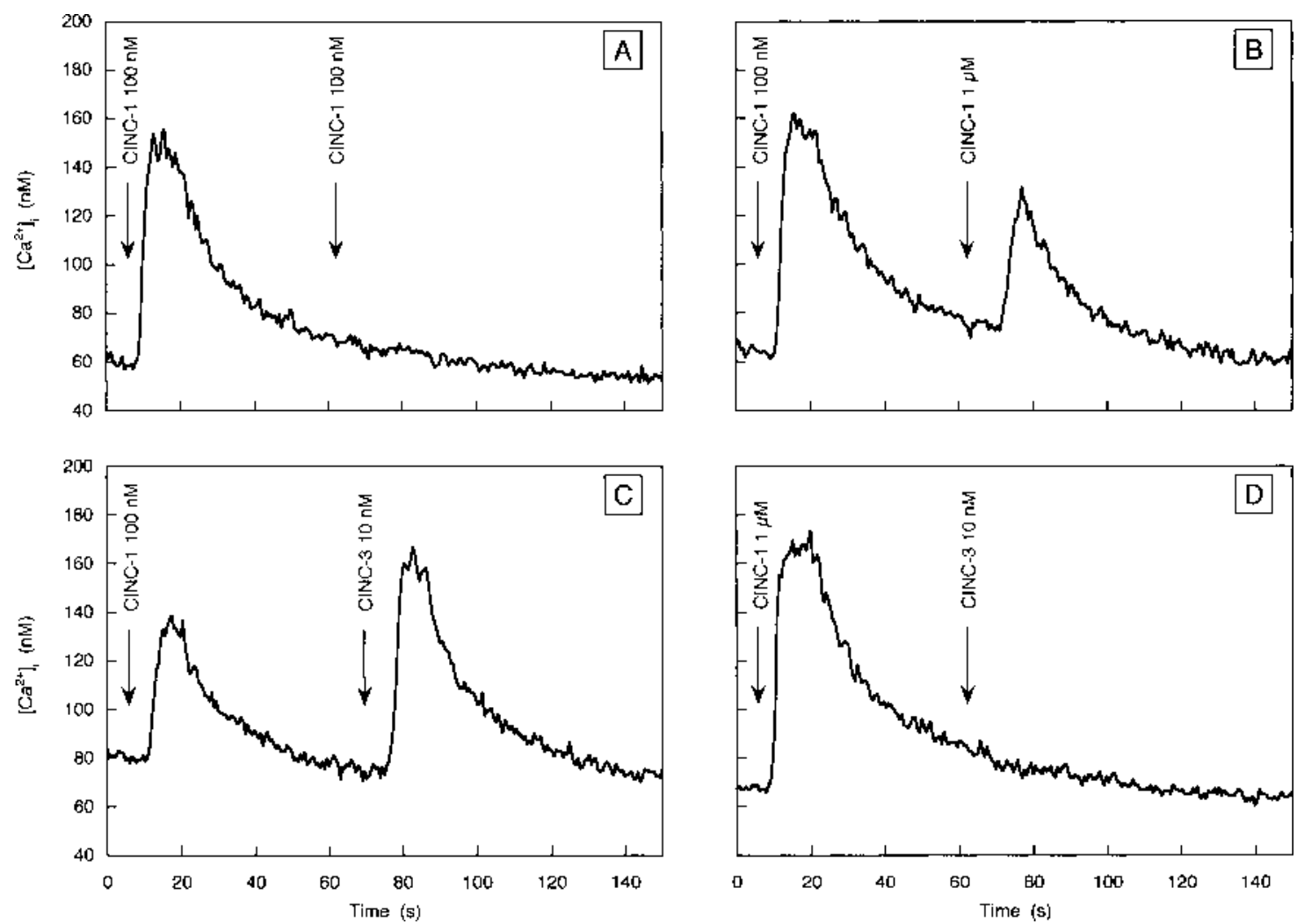

Fig. 1. Effects of Homologous and Heterologous Desensitization of Recombinant CINCs on Calcium Mobilization

Rat neutrophils were loaded with fura- 2 and stimulated by each CINC sequentially at the times indicated in the figure.

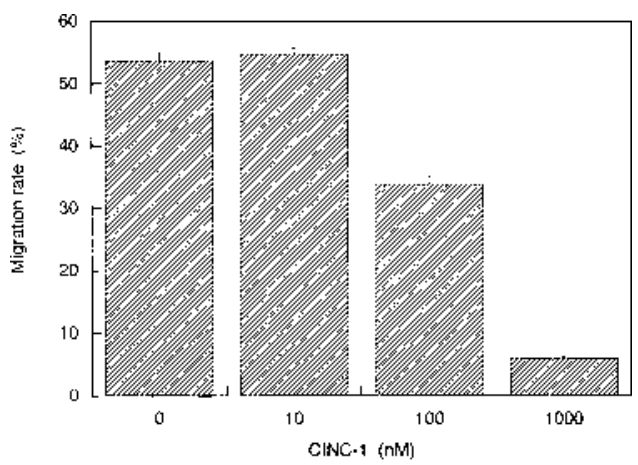

Fig. 2. Inhibition of CINC-3-Induced Neutrophil Chemotaxis by CINC-1

Rat neutrophils with CINC-1 were added to the upper wells of Boyden chambers and CINC-3 at a concentration of $10 \mathrm{~nm}$ was added to the lower wells. Migration of neutrophils was measured as described in Materials and Methods. Each point represents the mean \pm S.E.M. of six determinations.

at $10 \mathrm{~nm}$ induced calcium transients in neutrophils treated with CINC-1 $100 \mathrm{~nm}$ but not $1 \mu \mathrm{m}$ (Figs. 1C,D). Next, the effects of desensitization of CINCs on chemotactic activity were examined using Boyden chambers in vitro. The addition of CINC-1 to neutrophils in the upper wells of Boyden chambers inhibited the chemotactic activity of CINC-3 dose dependently (Fig. 2).

The effects of pertussis toxin on the biological activities of CINCs were measured to determine the differential regulation of these activities. Pertussis toxin treatment did not affect calcium mobilization of neutrophils induced by CINC-1 and CINC-3, whereas it inhibited the neutrophil chemotactic activity of CINC-1 and CINC-3 (Fig. 3).

\section{DISCUSSION}

In previous studies, we demonstrated the differences in neutrophil responses to CINC-1 and CINC-2 versus CINC$3{ }^{5,6)}$ CINC-3 at a concentration of $10 \mathrm{~nm}$ induced calcium mobilization to an extent similar to that induced by CINC-1 and CINC-2 at a concentration of $100 \mathrm{~nm}$. Stimulation of neutrophils with CINC-1 (100 nM) caused a loss of sensitivity to CINC-1 and CINC-2 (100 nM) but not to CINC-3 (10 nM). In contrast, CINC-3 (10 nM) desensitized calcium transients induced by all three types of CINCs. These responses were essentially identical to those observed in CXCR2-transfected HEK293 cells. $^{8)}$ In the present study, we found that the calcium mobilization induced by CINC-3 (10 nM) was inhibited completely by CINC-1 at a 100 -fold excess concentration $(1 \mu \mathrm{M})$ (Fig. 1). This result seems to reflect a 70 -fold lower affinity of CINC-1 to the receptor than CINC-3. ${ }^{7}$ On the other hand, CINC-3 showed chemotactic activity comparable with that of CINC-1 and CINC-2.5) The chemotactic activity of CINC-1 was inhibited completely by a 3 -fold excess of CINC-3, although the chemotactic activity of CINC-3 was not inhibited by a 3 -fold excess of CINC-1. ${ }^{6}$ This also appears to reflect the low affinity of CINC-1, because a 100 fold excess of CINC-1 desensitized the chemotaxis induced by CINC-3 (Fig. 2). Umland et al. reported that the relationship between the increase in intracellular calcium and the fraction of eotaxin receptor (CCR3) occupancy was linear in eosinophils, whereas a hyperbolic nonlinear relationship was observed between eosinophil chemotaxis and the receptor occupancy, suggesting that CCR3 interacted with G-proteins that were poorly coupled to the calcium response and effi- 

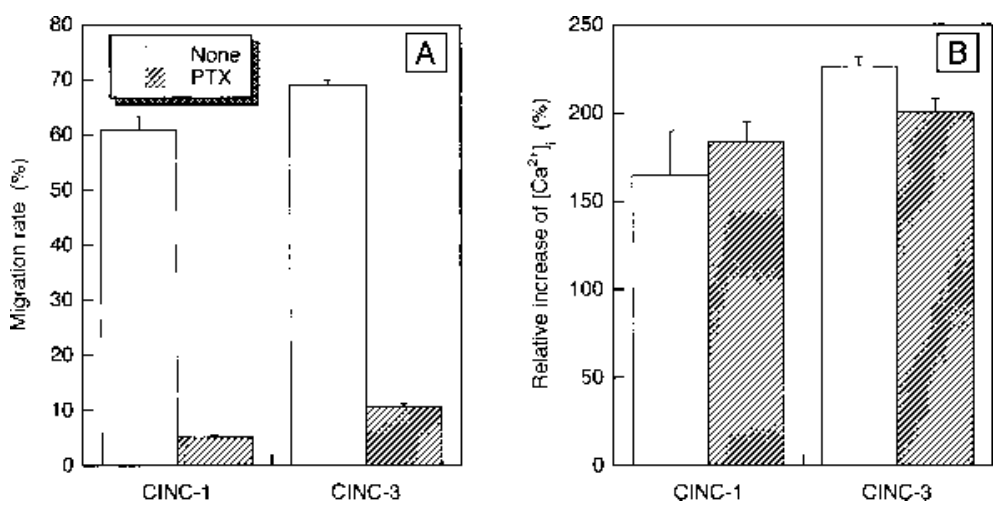

Fig. 3. Effect of Pertussis Toxin on Biological Activities of CINCs

Rat neutrophils were treated with pertussis toxin (PTX, $1 \mu \mathrm{g} / \mathrm{ml}$ ) at $37^{\circ} \mathrm{C}$ for $2 \mathrm{~h}$ and A) neutrophil chemotactic activity of CINCs, and B) CINCs-induced calcium transients were measured. Each column represents the mean \pm S.E.M. of four to six determinations.

ciently coupled to chemotaxis. ${ }^{10)}$ A similar explanation may be possible in the case of the CINC receptor CXCR2.

Formyl-methionyl-leucyl-phenylalanine, platelet-activating factor, and leukotriene B4 induced chemotaxis and calcium mobilization via pertussis toxin-sensitive G-protein in neutrophils. ${ }^{11-15)}$ However, others reported that platelet-activating factor and leukotriene B4 utilized an additional pertussis toxin-resistant mechanism for calcium mobilization. ${ }^{16)}$ Human neutrophils express two receptors, CXCR1 that binds IL-8 with high affinity and GRO $\alpha$ with low affinity, and CXCR2 showing high affinity to IL-8 and GRO $\alpha \cdot{ }^{1-20)}$ Although both receptors have been shown to be able to couple to both pertussis toxin-sensitive and -resistant G-proteins, ${ }^{21,22}$ they mediated IL-8-induced neutrophil chemotaxis via pertussis toxin-sensitive G-protein. ${ }^{23,24)}$ On the contrary, the calcium mobilization induced by IL- 8 at CXCR 2 was inhibited by pertussis toxin, but that induced by IL-8 at CXCR1 was not completely inhibited, suggesting the involvement of both pertussis toxin-sensitive and -resistant pathways in the signaling of CXCR1-elicited calcium mobilization. ${ }^{25)}$ CINC-1 and CINC-3-induced chemotaxis, but not calcium mobilization, was sensitive to inhibition by pertussis toxin (Fig. 3). The results were reproducible even when the same neutrophils treated with pertussis toxin were used for both assays. The different usage of G-proteins may come from the fact that rodent neutrophils have only one receptor, CXCR2. The results presented in this report suggest that CINCs induced neutrophil chemotaxis and calcium mobilization through distinct G-proteins with different efficiency.

\section{REFERENCES}

1) Nakagawa H., Komorita N., Shibata F., Ikesue A., Konishi K., Fujioka M., Kato H., Biochem. J., 301, 545-550 (1994).

2) Nakagawa H., Ando Y., Takano K., Sunada Y., Int. Arch. Allergy Immunol., 115, 137-143 (1998).
3) Takano K., Nakagawa H., Inflamm. Res., 50, 503-508 (2001).

4) Shibata F., Konishi K., Nakagawa H., Cytokine, 10, 169-174 (1998).

5) Shibata F., Konishi K., Kato H., Komorita N., al-Mokdad M., Fujioka M., Nakagawa H., Eur. J. Biochem., 231, 306-311 (1995).

6) al-Mokdad M., Shibata F., Nakagawa H., Biol. Pharm. Bull., 19, 879881 (1996).

7) Murakami K., Shibata F., al-Mokdad M., Nakagawa H., Ueno A., Kondo T., Biochem. Biophys. Res. Commun., 232, 562-567 (1997).

8) Shibata F., Konishi K., Nakagawa H., Cytokine, 12, 1368-1373 (2000).

9) Grynkiewicz G., Poenie M., Tsien R. Y., J. Biol. Chem., 260, 34403450 (1985).

10) Umland S. P., Wan Y., Shortall J., Shah H., Jakway J., Garlisi C. G., Tian F., Egan R. W., Billah M. M., J. Leukoc. Biol., 67, 441-447 (2000).

11) Verghese M. W., Charles L., Jakoi L., Dillon S. B., Snyderman R., J. Immunol., 138, $4374-4380$ (1987).

12) Goldman D. W., Chang F. H., Gifford L. A., Goetzl E. J., Bourne H. R., J. Exp. Med., 162, 145-156 (1985).

13) Becker E. L., Kermode J. C., Naccache P. H., Yassin R., Marsh M. L., Munoz J. J., Sha'afi R. I., J. Cell. Biol., 100, 1641-1646 (1985).

14) Lad P. M., Olson C. V., Grewal I. S., Scott S. J., Proc. Natl. Acad. Sci. U.S.A., 82, 8643-8647 (1985).

15) Molski T. F., Naccache P. H., Marsh M. L., Kermode J., Becker E. L., Sha'afi R. I., Biochem. Biophys. Res. Commun., 124, 644-650 (1984).

16) Haribabu B., Zhelev D. V., Pridgen B. C., Richardson R. M., Ali H., Snyderman R., J. Biol. Chem., 274, 37087-37092 (1999).

17) Ahuja S. K., Murphy P. M., J. Biol. Chem., 271, 20545-20550 (1996).

18) Holmes W. E., Lee J., Kuang W. J., Rice G. C., Wood W. I., Science, 253, 1278-1280 (1991).

19) Lee J., Horuk R., Rice G. C., Bennett G. L., Camerato T., Wood W., J. Biol. Chem., 267, 16283-16287 (1992).

20) Murphy P. M., Tiffany H. L., Science, 253, 1280-1283 (1991).

21) Wu D., LaRosa G. J., Simon M. I., Science, 261, 101-103 (1993).

22) Damaj B. B., McColl S. R., Mahana W., Crouch M. F., Naccache P. H., J. Biol. Chem., 271, 12783-12789 (1996).

23) Thelen M., Peveri P., Kernen P., von Tscharner V., Walz A., Baggiolini M., Faseb J., 2, 2702-2706 (1988).

24) Jones S. A., Moser B., Thelen M., FEBS Lett., 364, 211 -214 (1995).

25) Hall D. A., Beresford I. J., Browning C., Giles H., Br. J. Pharmacol., 126, 810-818 (1999). 